\title{
Multi-criteria decision making method based on Improved ELECTRE III model
}

\author{
Sun zhaoxu \\ School of Applied Mathematic \\ Central University of Finance and Economics \\ Beijing, China \\ E-mail: sunzhaoxu@163.com
}

\author{
Han min \\ School of Applied Sciences \\ Beijing University of Technology \\ Beijing, China \\ E-mail: hanm@bjut.edu.cn
}

\begin{abstract}
Aiming at the multi-criteria decision making problems in which the outranking relations between part of alternatives known by the decision maker, a method is proposed based on the improved ELECTRE III method. And the necessary and possible outranking relations can be achieved by the method. The advantage of the improved ELECTRE III method is introduced. Then the linear programming models based on the improved ELECTRE III method can be constructed to decide whether the necessary or possible outranking relation can be found between the alternatives. This approach overcomes the difficulty of inducing the criteria weight while using the valued outranking relation defined by the traditional ELECTRE III and refers to all the compatible weights and outranking relation threshold. At last, the approach is illustrated by a numerical example.
\end{abstract}

Keywords-Multi-criteria decision making; outranking relation; linear programming

\section{INTRODUCTION}

The Multi-Criteria Decision Aid (MCDA) has been one of the very fast growing areas of Operational Research (OR) during the two last decades. Multi-Criteria decision aiding often deals with ranking of many concrete alternatives from the best to the worst one, classifying alternatives from best to worst category or selecting the best alternatives based on multiple conflicting criteria ${ }^{1]}$. The MCDA is also concerned with theory and methodology that can treat complex problems encountered in management, business, engineering, science, and other areas of human activity.

There are two families of methodology for dealing with MCDA problem ${ }^{[2]}$. One is based on multi-criteria utility model. The weighted average method is a representative method of multi-criteria utility model. This method establishes utility function for each single criterion, and then the utility functions will be aggregated to an overall multiutility function according to the preferential information of the decision maker. At last, the alternatives can be ranked from best to worst based on the overall multi-utility function value. The other is the outranking methods. The well known ELECTRE (Elimination Et Choix Traduisant larealite) methods are the representative method. Outranking relation is the core idea of the outranking methods. The ELECTRE methods include ELECTRE I- ELECTRE V and ELECTRE TRI. ELECTRE III method is used frequently because this method is able to determine the valued outranking relation between alternatives. In this method, the preferential information will be given by the decision maker. This preferential information includes the criteria weight and threshold values of preference, indifference and veto for each criterion. Then the outranking relationship will be judged through the function of concordance and discordance. The method does not require the independence requirement of the evaluation criteria and can avoid the large difference ranking of the alternatives induced by small difference on some criteria. But this method cannot give the complete ranking of the alternatives because of the incomparable relation, and need more preferential information than the multi-utility model.

Whether the multi-utility method or the ELECTRE method, the preferential information should firstly given by decision maker. In many cases, the decision maker cannot give the exact values of the preferential information parameters, but can only give the preferential relations to parts of the all alternatives. These alternatives can be called training samples. Then an optimization model can be established to induce the exact values of the preferential parameters. The first robust ordinal regression method has been the generalization of the UTA method, called UTA GMS

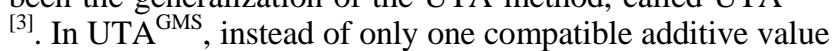
function composed of piecewise-linear marginal functions, all compatible additive value functions composed of general monotonic marginal value functions are taken into account. Further, this approach has been extended in the UTADIS ${ }^{\text {GMS }}$ method to deal with sorting problems ${ }^{[4],}$ and in ELECTRE $^{\mathrm{GKMS}}$, which is a general scheme implementing robust ordinal regression to outranking methods ${ }^{[5]}$. Robust ordinal regression has also been applied to preference model based on Choquet integral in order to handle interaction among criteria ${ }^{[6]}$.

This paper assumes that the preference, indifference and veto threshold are given by the decision maker. Whereas the weight of criterion and the threshold value of outranking relation are unknown. Based on the training alternatives' evaluation on each criterion and their outranking relations, linear programs can be built to get the possible and necessary outranking relations. This paper improves the literature ${ }^{[6]}$ in that only a specific evaluation criterion for the decision makers' preferences weight is concerned and literature ${ }^{[7]}$ on the aspect of difficulties to solve a mixed 0-1 integer linear programming problem. 


\section{THE IMPROVED ELECTRE III METHOD}

\section{A. Electre III method}

The valued outranking relation can be constructed by the Electre method which is proposed by Roy in 1978. A real value between 0 and 1 is conceived to indicate the preferential strength.

In this paper, we suppose that the criteria are all beneficial. The symbols $\boldsymbol{p}_{\boldsymbol{j}}, \boldsymbol{q}_{\boldsymbol{j}}, \boldsymbol{v}_{\boldsymbol{j}}(\boldsymbol{j}=1,2, \cdots, \boldsymbol{n})$ are denoted preference, indifference and veto threshold respectively. The evaluation of alternative $\boldsymbol{a}_{\boldsymbol{i}}$ on the criterion $\boldsymbol{g}_{\boldsymbol{j}}$ is denoted by $\boldsymbol{g}_{\boldsymbol{j}}\left(\boldsymbol{a}_{\boldsymbol{i}}\right)$. And then the valued outranking relation is constructed by the concordance and discordance index.

- The comprehensive concordance index

The comprehensive concordance index can be defined as follows:

$$
C\left(a_{i}, a_{k}\right)=\sum_{j=1}^{n} \omega_{j} c_{j}\left(a_{i}, a_{k}\right)
$$

Where

$$
c_{j}\left(a_{i}, a_{k}\right)=\left\{\begin{array}{rr}
0 & g_{j}\left(a_{i}\right)-g_{j}\left(a_{k}\right) \leq-p_{j} \\
1 & g_{j}\left(a_{i}\right)-g_{j}\left(a_{k}\right) \geq-q_{j}
\end{array}\right.
$$

$\omega_{j}$ is the weight of the criterion $\boldsymbol{g}_{j}$, and $C\left(a_{i}, a_{k}\right)$ is the comprehensive concordance index to support the assertion that " $a_{i}$ outranks $a_{k}$ ”.

- $\quad$ The discordance index

$$
\boldsymbol{d}_{j}\left(a_{i}, a_{k}\right)=\left\{\begin{array}{lc}
0 & g_{j}\left(a_{k}\right)-g_{j}\left(a_{i}\right) \leq p_{j} \\
1 & g_{j}\left(a_{k}\right)-g_{j}\left(a_{i}\right) \geq v_{j} \\
\left(g_{j}\left(a_{k}\right)-g_{j}\left(a_{i}\right)-p_{j}\right) /\left(v_{j}-p_{j}\right) & \text { otherwis }
\end{array}\right.
$$

- The valued outranking relation index

$$
S\left(a_{i}, a_{k}\right)=C\left(a_{i}, a_{k}\right) \cdot \prod_{j=1}^{n} N D_{j}\left(a_{i}, a_{k}\right)
$$

Where

$$
N D_{j}\left(a_{i}, a_{k}\right)=\min \left(1, \frac{1-d_{j}\left(a_{i}, a_{k}\right)}{1-C\left(a_{i}, a_{k}\right)}\right)
$$

From formula (4) and (5), we can see that the discordance index will affect the valued outranking relation if the relation of $\boldsymbol{d}_{\boldsymbol{j}}\left(\boldsymbol{a}_{\boldsymbol{i}}, \boldsymbol{a}_{\boldsymbol{k}}\right)>\boldsymbol{C}\left(\boldsymbol{a}_{\boldsymbol{i}}, \boldsymbol{a}_{\boldsymbol{k}}\right)$ is true.

- $\quad$ The outranking relation index

We use $\lambda \in[0.5,1]$ to denote the threshold of outranking relation. If outranking relation index is greater than the threshold of outranking relation, then the assertion that " $a_{i}$ outranks $a_{k}$ ” is true. Otherwise the assertion will be conceived false.

\section{B. The improved Electre III method}

From the equation (4), we can see that the valued outranking relation include the unknown weight of criterion when the decision maker cannot give the preference information about the criteria. Thus the linear programming model cannot be constructed to induce the weight of criteria. So the comprehensive concordance index $\boldsymbol{C}\left(\boldsymbol{a}_{\boldsymbol{i}}, \boldsymbol{a}_{\boldsymbol{k}}\right)$ can be replaced by constant $\alpha_{j}$ and the new $N D_{j}^{\prime}\left(\boldsymbol{a}_{\boldsymbol{i}}, \boldsymbol{a}_{\boldsymbol{k}}\right)$ can be built by the new defined $\boldsymbol{d}_{j}^{\prime}\left(\boldsymbol{a}_{\boldsymbol{i}}, \boldsymbol{a}_{\boldsymbol{k}}\right)$.

$$
\boldsymbol{d}_{j}\left(a_{i}, a_{k}\right)=\left\{\begin{array}{lr}
0 & g_{j}\left(a_{k}\right)-g_{j}\left(a_{i}\right) \leq u_{j} \\
1 & g_{j}\left(a_{k}\right)-g_{j}\left(a_{i}\right) \geq v_{j} \\
\left(g_{j}\left(a_{k}\right)-g_{j}\left(a_{i}\right)-u_{j}\right) /\left(v_{j}-u_{j}\right) & \text { otherw }
\end{array}\right.
$$

Where $\boldsymbol{u}_{j}=\boldsymbol{p}_{j}+0.75\left(\boldsymbol{v}_{j}-\boldsymbol{u}_{j}\right)$.

Then the new $\boldsymbol{N} \boldsymbol{D}_{\boldsymbol{j}}^{\prime}\left(\boldsymbol{a}_{\boldsymbol{i}}, \boldsymbol{a}_{\boldsymbol{k}}\right)$ and $\tilde{S}\left(a_{i}, a_{k}\right)$ are defined as follows:

$$
\begin{gathered}
N D_{j}^{\prime}\left(a_{i}, a_{k}\right)=1-d_{j}^{\prime}\left(a_{i}, a_{k}\right) \\
\tilde{S}\left(a_{i}, a_{k}\right)=C\left(a_{i}, a_{k}\right) \cdot \prod_{j=1}^{n} N D_{j}^{\prime}\left(a_{i}, a_{k}\right)
\end{gathered}
$$

\section{THE LINIEAR PROGRAMMING MODEL BASED ON THE} PREFERENCE OF DECISION MAKER

We use the symbol of $\boldsymbol{A}^{\boldsymbol{R}}$ to indicate the alternatives that decision maker can give the outranking relation. If the decision maker considers that alternative $\boldsymbol{a}$ outranking alternative $\boldsymbol{b}$, then we use the symbol $\boldsymbol{a} \boldsymbol{S}_{\boldsymbol{D M}} \boldsymbol{b}$. The following linear programming can be constructed as following:

$\max \varepsilon$

s.t $\left.\tilde{S}(a, b)=C(a, b) \cdot \prod_{j=1}^{n} N \dot{D}(a, b)=C(a, b) \cdot \prod_{j=1}^{n} 1-d_{j}(a, b)\right) \geq \lambda$

foralla, $b \in A^{R}, a S_{b M} b$

$\tilde{S}(\boldsymbol{a}, \boldsymbol{b})=C(\boldsymbol{a}, \boldsymbol{b}) \cdot \prod_{j=1}^{n} N \dot{\not}(\boldsymbol{a}, \boldsymbol{b})=C(\boldsymbol{a}, \boldsymbol{b}) \cdot \prod_{j=1}^{n}\left(1-d_{j}(\boldsymbol{a}, \boldsymbol{b})\right) \leq \lambda-\varepsilon$

$$
\text { forall } a, b \in A^{R}, a S_{D M}^{C} p
$$$$
\omega_{j} \geq 0, \sum_{j=1}^{n} \omega_{j}=1
$$

$0.5 \leq \lambda \leq 1$

The constraint conditions in model (9) is dedicated by $E^{A^{R}}$.If the feasible area of model (9) is not empty and the optimization value is greater than 0 , then there are at least one solution correspond to decision maker' s preference. 
In order to get the outranking relation among alternatives, we construct the following linear programming model:

$$
\begin{array}{cc}
\max & \varepsilon \\
\text { s.t } E^{A^{R}} \text { 且 } \tilde{S}(a, b) \leq \lambda-\varepsilon
\end{array}
$$

If the problem has no feasible solution or the optimization value is lower than 0 . We can conclude that all the parameters of decision maker's preference can induce the outranking relation indicated by the assertion " $\boldsymbol{a}$ outranking alternative $\boldsymbol{b}$ ”. We define such outranking relation as necessary outranking relation.

In contrast to the necessary outranking relation, we also define another outranking relation: possible outranking relation.

$$
\begin{aligned}
& \max \varepsilon \\
& \text { s.t } E^{A^{R}} \text { 且 } \tilde{S}(a, b) \geq \lambda
\end{aligned}
$$

If the feasible solution of problem (11) are not empty and the optimization value is greater than 1 , then we can conclude that at least one set parameter of decision maker's preference can induce the outranking relation indicated by the assertion " $\boldsymbol{a}$ outranking alternative $\boldsymbol{b}$ ". We define such outranking relation as possible outranking relation.

\section{AN NUMERICAL EXAMPLE}

We will propose a simple numerical example to illustrate the method proposed by this paper.

There are six alternatives which are evaluated by 3 criteria that are profit, cost and safety. The unit of criteria profit and cost is ten thousands. The safety criterion is evaluated by expert. Table 1 shows the evaluation of alternatives.

\begin{tabular}{|c|c|c|c|}
\hline TABLE1 THE EVALUATION OF ALTERNATIVES \\
\hline Alternatives & profit & cost & safety \\
\hline 1 & 276 & 136 & 80 \\
\hline 2 & 256 & 124 & 85 \\
\hline 3 & 325 & 143 & 75 \\
\hline 4 & 312 & 132 & 90 \\
\hline 5 & 295 & 121 & 85 \\
\hline 6 & 286 & 152 & 80 \\
\hline
\end{tabular}

Table 2 gives the threshold value of preference, indifference and veto.

TABLE 2 THE PREFERENCE,INDIFFERENCE AND VETO THRESHOLD

\begin{tabular}{|c|c|c|c|}
\hline \multicolumn{2}{|c|}{ TABLE 2 THE PREFERENCE,INDIFFERENCE AND VETO THRESHOLD } \\
\hline Criterion & $\begin{array}{c}\text { Preference } \\
\text { threshold }\end{array}$ & $\begin{array}{c}\text { Indiffernce } \\
\text { threshold }\end{array}$ & Veto threshold \\
\hline Profit & 36 & 28 & 50 \\
\hline Cost & 16 & 13 & 21 \\
\hline Safety & 11 & 6 & 16 \\
\hline
\end{tabular}

According to the decision maker's preference, alternative 1 outranks alternatives 2.For the alternative 3,4,5,6 ,we use the method proposed by the above method and get the following possible and necessary outranking relationt: a) Alternative ${ }_{4} \boldsymbol{S}^{\boldsymbol{P}}$ Alternative 3; Alternative ${ }_{4} \boldsymbol{S}^{\boldsymbol{P}}$ Alternative 5; Alternative $5^{\boldsymbol{S}^{\boldsymbol{P}}}$ Alternative 3; Alternative ${ }_{4} \boldsymbol{S}^{\boldsymbol{P}}$ Alternative 6; Alternative $5 \boldsymbol{S}^{\boldsymbol{P}}$ Alternative 6。

b) Alternative $4 \boldsymbol{S}^{N}$ alternative 6 ; alternative ${ }_{5} S^{N}$ alternative 6。

\section{CONCLUSION}

In this paper, with the aiming at the multi-criteria decision making problems in which the outranking relations between part of alternatives known by the decision maker, a method is proposed based on the improved ELECTRE III method. And the necessary and possible outranking relations can be achieved by the method. The advantage of the improved ELECTRE III method is introduced. Then the linear programming models based on the improved ELECTRE III method can be constructed to decide whether the necessary or possible outranking relation can be found between the alternatives. This approach overcomes the difficulty of inducing the criteria weight while using the valued outranking relation defined by the traditional ELECTRE III and refers to all the compatible weights and outranking relation threshold. At last, the approach is illustrated by a numerical example.

\section{ACKNOWLEDGMENT}

This paper is supported by the 211 Research Funds of Central University of Finance and Economics and Basic Research Foundations of Beijing University of Technology (X4006012201101)

\section{REFERENCES}

[1] Chen Ting. Decision Making Analysis[M].Beijing: Science Press, 1987

[2] Roy. B. Multi-criteria Methodology for Decision Aiding [M]. Dordrecht: Kluwer Academic Publishers,1996.

[3] S.Greco, V.Mousseau, R.Slowinski.Ordinal regression revisited Multiple criteria ranking using a set of additive value functions[J].European Journal of Operational Research,2008,191:416-436.

[4] P.Fortemps,M.Pirlot. ACUTA:A novel method for eliciting additive value functions on the basis of holistic preference statements[J].European Journal of Operational Research,2010,206: 435-444.

[5] S.Greco, V.Mousseau,R.Slowinski. Multiple criteria sorting with a set of additive value functions[J].European Journal of Operational Research,2010,207:1455-1470.

[6] S.Angilella,S.Greco, B.Matarazzo. Non-additive robust ordinal regression A multiple criteria decision model based on the Choquet integral[J].European Journal of Operational Research,2010,201:277288.G.Bous a,

[7] S.Greco, V.Mousseau,R.Slowinski. ELECTRE ${ }^{\mathrm{GKMS}}$ :Robust ordinal regression for outranking methods[J].European Journal of Operational Research,2011,214:118-135.

[8] Mousseau.V.Valued outranking relation in ELECTRE III providing manageable disaggregation procedures[J].European Journal of Operational Research,2004,158 (3) : 528-547. 